Prozedere bei

Doppelklappenerkrankungen

Das Prozedere bei Doppelklappenerkrankungen ist davon abhängig, ob ein Elektiveingriff indiziert ist oder ob es sich um eine Notfallindikation z. B. wegen Dekompensation handelt. Zudem ist zu klären, ob eine Endokarditis vorliegt. Schließlich werden morphologische/ technische Aspekte im Herzteam erörtert. Bei der Mitralklappe geht es neben der Frage nach Verkalkung und Morphologie darum, ob die Klappe ein ClipVerfahren zulässt.

Nach Ansicht von Walther steht das Herzteam bei folgenden Konstellationen vor der Frage, ob chirurgisch oder interventionell vorgegangen werden soll oder ob beide im Hybrid-OP zugreifen sollen, etwa mit TAVI und konventioneller Mitralisreparatur: Bei Vorliegen einer Aortenklappe mit intermediärem oder hohem Risiko bei gleichzeitiger Mitralklappe mit sekundärem, funktionellen Defekt.

Optimal wäre es dem Herzchirurgen zufolge, wenn solche Doppelklappeneingriffe in einem Herzklappen-Zentrum vorgenommen würden, wie sie derzeit auf europäischer Ebene zwischen den europäischen Gesellschaften für ThoraxChirurgie (EACTS) und Kardiologie
(ESC) vorgeschlagen und auch von der DGK diskutiert werden.

Er fand dafür einen Vergleich aus dem Rennsport. Der Formel-1-Pilot ist auf ein gut trainiertes Team mit unterschiedlichsten Fähigkeiten angewiesen. Die strukturierte Kollaboration ermöglicht erst den Erfolg. Ähnlich ist in einem Herzklappenzentrum ein Herzteam unerlässlich, soll das Optimum für den Patienten herausgeholt werden.

Den Teamgedanken von Walther honorierten die Zuhörer. In der TED-Abstimmung holte er in der Zustimmung von 30 auf $56 \%$ auf.

Dr.med. Jochen Aumiller

\section{CASTLE-AF - ein Meilenstein für die Rhythmologie}

\begin{abstract}
Für Patienten mit Vorhofflimmern und Herzschwäche hat sich die Katheterablation in der CASTLE-AF-Studie als prognostisch von Vorteil erwiesen - für den DGK-Tagungsleiter ist dieses Ergebnis ein Durchbruch.
\end{abstract}

—ür die Rhythmologie war es eine Zä- sur, wie der Tagungsleiter Prof. Dietmar Bänsch vom KMG Klinikum Güstrow betonte. Erst die CASTLE-AF-Studie hat belegt, dass die Katheterablation bei Vorhofflimmern die Sterblichkeit dramatisch verringert. Bislang empfahl man diese Elektrotherapie vor allem wegen der symptomatischen Besserung, die der Wirkung von Antiarrhythmika deutlich überlegen war.

In die CASTLE-AF-Studie wurden 397 Patienten aus 33 Zentren in Europa, Australien und in den USA mit Vorhofflimmern und Herzschwäche (Auswurffraktion $<35 \%$, alle mit implantierbarem Kardioverter-Defibrillator) randomisiert entweder mit Medikamenten oder einer Ablation behandelt.

Nach einer medianen Beobachtungszeit von 37,8 Monaten war in der Ablations-Gruppe sowohl die Gesamtmortalität geringer als auch die kardiovaskuläre Letalität wie auch die Hospitalisierungen wegen Herzinsuffizienz. Der primäre Endpunkt - die Gesamtmortalität und ungeplante Hospitalisierungen wegen dekompensierter Herzinsuffizienz - wurde durch die Ablation im Vergleich zur medikamentösen Therapie um $38 \%$ gesenkt
( 28,5 vs. $44,6 \%)$ - ein hochsignifikantes Ergebnis.

\section{„Brauchen abladierte Patienten noch eine Antikoagulation?"}

Auf eine wichtige praktische Frage machte PD Dr. Christopher Piorkowski,

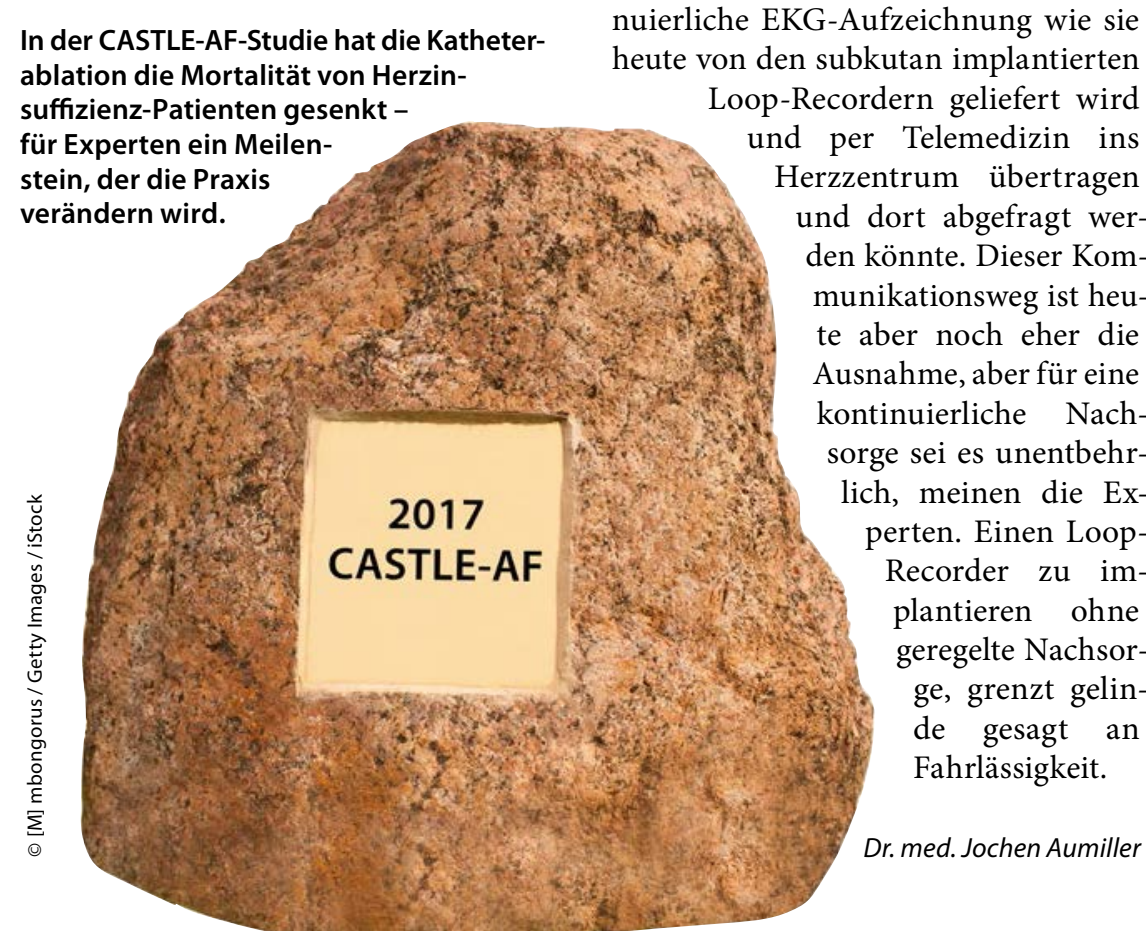

Herzzentrum Dresden, aufmerksam: Benötigen erfolgreich abladierte Patienten mit Herzinsuffizienz grundsätzlich eine Fortführung der Antikoagulation? „Im klinischen Alltag wird diese Frage häufig pragmatisch und im Sinne einer individuellen Risikoabschätzung gehandhabt, um Patienten nicht langfristig einer gerinnungshemmenden Therapie mit den zwangsläufigen Blutungsrisiken auszusetzen. In Zukunft brauchen wir dafür aber prospektive Evidenz."

$\mathrm{Ob}$ und wie lange antikoaguliert werden soll, hängt wesentlich von der Schwere der Vorhofflimmerlast ab. Um sie abzuschätzen, braucht es eine kontinuierliche EKG-Aufzeichnung wie sie heute von den subkutan implantierten oop-Recordern geliefert wird per Telemedizin ins ubertragen den könnte. Dieser Kommunikationsweg ist heute aber noch eher die fusnahme, aber für eine ch, meinen die ExRecorder $\mathrm{zu}$ implantieren ohne pachsore, grenzt gelinde gesagt an Fahrlässigkeit. 